\title{
An Empirical Evaluation of Graphical Usable Interface on Mobile Chat
}

\author{
Victoria Yee Siew Yen ${ }^{1}$ and Daniel Su Kuen Seong ${ }^{2}$ \\ ${ }^{1}$ Financial Services, Accenture, Malaysia \\ victoria.yeedaccenture.com \\ ${ }^{2}$ The University of Nottingham, Malasysia \\ daniel.su@nottingham. edu.my
}

\begin{abstract}
Current text-based mobile group chatting systems hinder navigation ease through long chat archive in a limited screen display. Moreover, tracking messages sent by specific chatter is cumbersome and time consuming. Hence, graphical-based usable interface that aids navigation and message tracking through minimal key-pressed and enhances user expression via avatars employment is proposed. The research outcomes typified that there was significant linear relationship between user interface and usability on text-based and graphical-based usable interface on mobile chat. Moreover, the experimental evaluation results indicated that text-based usability could be improved by creating interface that encourages usages whereas the graphical-based usable mobile chat is augmented by crafting user friendly interface that enhances user satisfaction, encourages usages and promotes navigation ease. The empirical findings and results exemplified that the potential use of graphical-based usable mobile chat as substitution to the text-based that presently has poor reception and is under utilised in commercial arena.
\end{abstract}

\section{Introduction}

Communication is a salient part of life and is used to convey social presence, augment social bonding and relay information. The advent of networked computer has inadvertently supported this notion by facilitating communication services such as electronic mail, instant messages, chat and video conferencing to be incorporated into existing computing technology. In effect, communication system that congregates users in the virtual context despite their physical proximity has been widely adopted. The emerging trend of migrating commercially successful desktop applications into mobile environment is the heart of numerous mobile researchers. Generic categorisation of the offered mobile services has been developed and classified into content, commerce and communication [1]. Hence, mobile chat is intended to be the core communication of the study in this paper.

Vronay et al. [2] has conducted an in-depth investigation on chat system and defined chat as "two to twenty or more people who appear together on a common channel of communication known as chat room." This vague explanation merely stresses on the social gathering in a public chatting space without emphasising on actual engagement of conversation and nature of communication. 
In the effort to clarify terminology, ISO/IEC JTC1 SC36 [3] has delineated chat as "a form of synchronous interactive online typewritten communication allowing users to engage in text-message conferencing. Chat is also used for private communications between a subset of participants." The definition neither associates to usability nor conceptualise nonverbal cues such as body language and facial expression that is crucial in conversation.

Evidently, a novel description that facilitates expressive communication is needed. Thus, chat is characterised as a virtual congregation of two or more participants in synchronous communication mode that enable articulate interaction via texts, graphics and images in both private and public chat rooms.

In the exertion to support mobility, the prerequisite for the design of mobile devices is the diminutive figure of the gadget. Consequently, display screen is devised to be small [4] and effective presentation of huge and diversified amount of information through the small window posed a dilemma. Furthermore, attention has been given to the processing requirement of mobile programs to ensure low utilisation of the limited processing capacity [4]. In respect of the restricted storage and memory, the similar consideration should be granted to avoid high consumption of storage and memory. Additionally, the dynamic nature of wireless connectivity introduces delay and hinders smooth interaction among the users.

Due to the physical limitation of mobile devices, maximum displayable number of turns at any one time is significantly smaller than stationary chat system which subsequently hampers usability. Contrary to immobile chat system which archives history locally, the similar situation does not apply to mobile chat system due to the inadequate storage capacity. In addition, mobile device limitations impeded mass gathering and reduced the optimal number of users logged into a chat room. Moreover, navigation via both mobile and stationary chat systems differs due to the heterogeneous input mechanisms. In the light of the variation, navigation via mobile keypad could be unwieldy and thus, requires special attention in the design of the system.

Generic problems existed between mobile and stationary chat system could be resolved by improving and enhancing the user interface of the system. In particular, chat system does not facilitate recognition of previously chatted users and the particulars related to the users [2]. Consequently, tension between users might be created as offended party viewed negatively about the forgetful party. Furthermore, individual presence of users who are not actively participating in the chat room is not conveyed appropriately [2]. Indication of the user status such as "away" and "busy" should be revealed to inform the availability of users. Other overriding theme to uncover is the inability of chat system to express complex human embodiment that is crucial in daily interaction. Thus, this paper aims to statistically evaluate text-based and graphicalbased usable interface on mobile chat and draw these observations and findings to uncover driving factors that would enhance mobile chat usability.

\section{Literature Review}

Burak and Sharon [5] have conducted an extensive usage study on mobile services, named FriendZone. They have concluded that chat application is not suitable for mobile phones due to the small screen display and cumbersome keypad that does not 
encourage fast text entry. In opposition, Grinter and Eldridge [6], [7] argued and confirmed that teenagers have flexibility in adapting to the physical restriction of the mobile devices for efficient text entry which consequently become primary motivation for the mobile chat usage. Hence, prior to the design of mobile chat system, indepth knowledge with regards to habits and preferences of potential users should be gained to increase usability of the system and enrich user experience.

Vronay et al. [2] proposed a novel interface that is efficient to cater for high amount of users. Nonetheless, porting such design to small screen space is inappropriate as it cluttered the screen display easily. Nevertheless, we believe that conceptualising interactions in horizontal course facilitates the efficient tracking of turns in a limited screen display.

The minimalist approach adopted by Viegas and Donath [8], [9] in Chat Circles series manipulated unique colours to represent individual presence. Colours are perceived differently according to cultural and demographic background. For instance, green is perceived as unlucky in Britain and Ireland, and attire of green must be avoided in weddings [10]. Conversely, green represents longevity and life to Chinese in conjunction with their preference to green jade or nephrite [10]. Direct inference from this finding to chatting system is users whom selected biased colour to a particular community might not be welcomed and even treated harshly. Furthermore, favourable colours might be highly utilised resulting in ambiguous representation of individual identity due to the small palette of colour. By observing these differences, conclusive statement could be drawn that usages of colour for identity representation is highly controversial and critical. Viegas and Donath [9] have integrated background images which served as shared contexts to promote congregation and encouraged conversation around the images. Amin et al. [11] have supported this notion by stating that shared awareness of the settings, objects and influences in the surrounding context augment the communication and minimise misapprehension. Therefore, usage of background images should be employed as it facilitates interaction and introduces topics for discussions resulting in a usable system.

Chat system that depends on text as the sole communicative element lacks the facility to convey nonverbal cues presented in face-to-face communication. Thus, numerous investigations and experiments [11], [12], [13], [14] have conducted to enrich the user experience by integrating behavioural expressiveness such as angry and upset into avatars and emoticon. Amin et al. [11] have recommended an extension to Short Message Service (SMS) named SenseMS which allows emotion and context to be readily perceived prior to the viewing of message content to aid understanding. While this design is developed for asynchronous nature of communication, repeated display of emotional status prior to each turn in real time environment introduces redundancy and attenuates the usability of the system.

\section{Research Design and Methodology}

\subsection{Overview}

The target sample chosen was teenagers as recorded to be the forerunner of mobile chat with intention to enhance the viability of this study. There were 4 surveys 
carried out in different time and locations to facilitate and coordinate the different time availability of the test subjects. The chosen local mobile chat service was Maxis SMS Chat which uses SMS as the underlying communication protocol for messages exchange.

Henceforth, Maxis SMS Chat was refereed as text-based mobile chat system (TMC) and our proposed system as graphical-based usable mobile chat system (GMC). Distinct difference between TMC and GMC was the user interface design of TMC utilised text as the sole element while GMC employed a myriad of text and graphics, and appropriate avatar and colours in the interface design as illustrated in Fig. 1. This difference could be harnessed by investigating user reception and perception on both text and graphical-based usable interfaces of mobile chat.

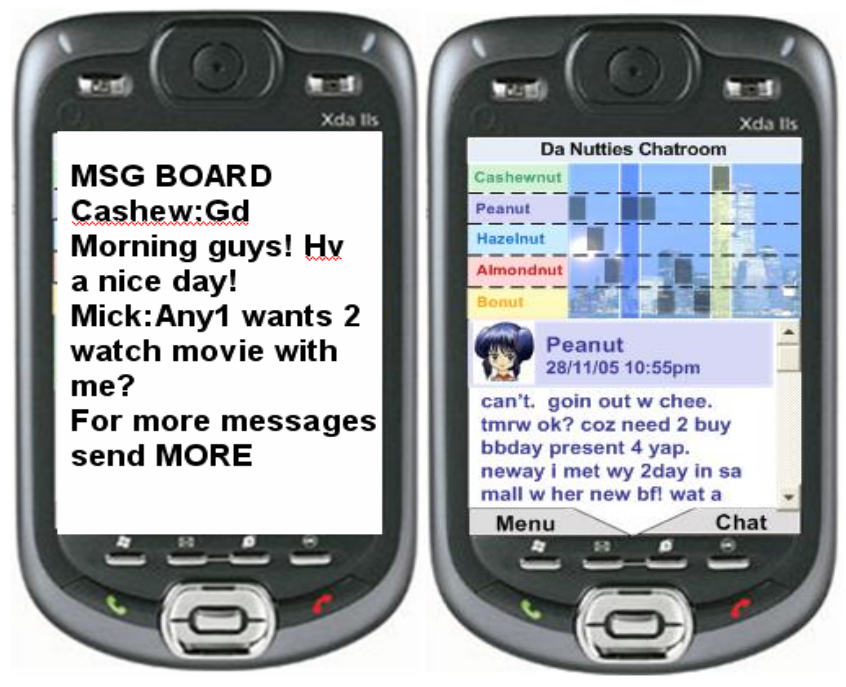

Fig. 1. User interfaces of TMC and GMC respectively

\subsection{Demography of Test Subjects}

A total of 53 test subjects involved in this experiment with demographic background that included different gender, education level and age. The test subjects were within the age range of 15 to 21 which reflected the age classification of teenagers. In all, 25 females and 28 males have participated in the survey. As a measure to effectively segregate the sample based on the education level, 27 test subjects were selected from The University of Nottingham, Malaysia, and 26 from a local secondary school in the district of Klang Valley, Malaysia.

\subsection{Materials Used}

There were 2 additional materials enclosed with the questionnaire distributed to the test subjects: the user documentation of Maxis SMS Chat which intended to assist the 
understanding of the existing commands for service activation and utilisation; whereas the supplementary material served to define terminology found in the questionnaire that might be unfamiliar to teenagers with lower computing knowledge such as "user interface", "usability", "navigate" and so forth.

\subsection{Experiments}

The experiments for both groups took place in a control environment for 1.5 hours. The procedures began with a briefing, followed by testing and evaluation of TMC and GMC and ended with demonstration and assessment of TMC and GMC.

\section{Findings, Results and Discussions}

Prior to data analysis, reliability of the instrument was assessed to ensure the internal consistency holds. Generally, alpha value that exceeds 0.60 is accepted to have an internally consistent construct [15]. Both TMC and GMC constructs were internally consistent with coefficient values 0.610 and 0.616 respectively.

\subsection{Hypotheses}

There were 2 hypotheses formulated as initial presumptions on the relationship between user interface and usability. Table 1 details the hypotheses and the corresponding descriptions. All the hypotheses were formulated to test and evaluate at $95 \%$ (0.05) significant value as to fulfil the research objectives.

Table 1. Research hypotheses statement

\begin{tabular}{ll}
\hline $\mathrm{H}_{\#}$ & Narration \\
\hline $\mathrm{H}_{1}$ & There is a significant linear relationship between user interface and usability of TMC \\
$\mathrm{H}_{2}$ & There is a significant linear relationship between user interface and usability of GMC \\
\hline
\end{tabular}

\subsection{Background of Test Subjects}

As coincide with the research carried out by Grinter and Eldridge [6], [7], SMS is the most preferred chatting tool among teenagers, which in this case also applied to Malaysian teenagers. Only 3 test subjects (5.7\%) have not communicated via SMS while more than half $(50.9 \%)$ used the service actively which were more than 20 times per week.

Multimedia Messaging Service (MMS) as anticipated and consistent with study reported by Amin et al. [11], yielding low usage as $73.6 \%$ of the sample has not used the service before. This is not surprising as the service is restricted to certain mobile devices and the cost associated to each is 2.5 times more than per SMS in Malaysia [16]. On the static Internet platform, 75.5\% of the sample has used online chat service which corroborated to the findings from Pew Internet and American Life Project 
[17] with a marginal difference of $0.5 \%$. Mobile group chatting services scored an extremely low usage with a staggering $83 \%$ of the test subjects have not used the service. The low usage is expected as mobile group chatting services are not well advertised and developed in Malaysia.

\subsection{Hypotheses Evaluation}

Null hypotheses were statistically tested to determine the trueness of the claims. In the case of hypotheses $\mathrm{H}_{1}$ and $\mathrm{H}_{2}$, multiple regression analyses were conducted to test the trueness of the hypotheses with usability as the dependent variable and user interface as independent variables.

\subsection{Relationship Between User Interface and Usability of TMC}

Research outcomes of the multiple regression analysis indicated there was a significant relationship $(\mathrm{p}=0.003)$ between the user interface and usability of TMC. Based on the beta coefficient, we observed that there existed a positive linear relationship $(\beta=0.395)$ between the user interface and usability of TMC in which the enhancement to the user interface that subsequently encourages usages of the service would increase the usability of TMC. This relationship could be simplified via the equation:

Usability of $\mathrm{TMC}=($ User interface that encourages usage $) \times 0.395$. [18]

The beta coefficient is essential to determine the relative absolute magnitude in predicting the usability of TMC. Research findings signified that the increased of 1 unit on TMC usability requires 0.395 increased of user interface that encourages usages. Although user interface assessments are qualitative could not be effectively represented with real numbers or figures, the beta coefficient provided insight as to the magnitude of influence the variable has against the usability of TMC. Thus, the results denoted that the effort to enhance the usability on text-based mobile chat service should be placed in ensuring the user interface that encourages usages. Other variables vital to usability such as navigation, user satisfaction, and effectively were not significantly linear correlated plausibly because the importance on improving user interface for usages has overshadowed other usability aspects. Based on the analysis, $\mathrm{H}_{1}$ experienced a rejection of null hypothesis.

\subsection{Relationship Between User Interface and Usability of GMC}

It is evident through the significant values that there were strong correlations among the user friendliness of interface $(\mathrm{p}=0.001)$, user satisfaction induced by user interface $(\mathrm{p}=0.007)$ and usages promoted by user interface $(\mathrm{p}=0.011)$ on the usability of GMC. The evaluation of the beta coefficients has highlighted that all the linear regression has positive relationship with usability.

Usability of GMC $=($ User friendliness of interface $) \times 0.402+($ Satisfaction enhanced by user interface) $\mathrm{x} 0.320+$ (User interface that encourages usage) $\mathrm{x}$

$$
0.303+(\text { Navigation ease of user interface) } \mathrm{x} 0.171 \text {. [18] }
$$

We could safely conclude that user friendliness of interface that enhances user satisfaction and encourages usages possess relatively strong magnitudes in the 
correlations with usability on GMC. The user friendliness of interface has the highest absolute value $(\beta=0.402)$ as compared to other variables significantly typified that it correlated strongly with the usability. The equation highlighted that 1 unit increased of GMC usability requires 0.402 increased of user friendly interface, 0.320 of satisfied user interface, and 0.303 of user interface that encouraged usage, and 0.171 of navigation ease. Evidently, work to improve the usability of GMC should be placed firstly in creating user friendly interface followed by other variables in the order of their beta coefficient values. Hence, $\mathrm{H}_{2}$ manifested a rejection of null hypothesis.

\subsection{Results and Discussions}

Generally, the evaluation for each attribute in GMC surpassed that of TMC considerably with the lower mean difference being 2.42 or a staggering $48.4 \%$ rating difference between both services. These figures added the credential of graphical-based usable interface being a better design solution than text-based user interface on mobile chat in every aspect of user interface and usability that were being assessed. The findings and results supported the main distinction between text-based and graphicalbased usable mobile chat was attributed to the navigation ease with GMC scoring favourably high $(\bar{x}=4.47)$ as opposed to TMC $(\bar{x}=1.45)$. Additionally, the user friendliness of interface $(\bar{x}=4.51)$, learnability $(\bar{x}=4.43)$, well integration of functions $(\bar{x}=4.42)$, consistency $(\bar{x}=4.38)$ and simplicity $(\bar{x}=4.38)$ were the highest rated attributes for GMC evaluation. These factors are undeniably the key advantages of employing graphical-based usable design on mobile chat. In contrast, the experimental evaluation results denoted that inconsistency $(\bar{x}=1.42)$, difficulty in navigation $(\bar{x}=1.45)$, complexity $(\bar{x}=1.49)$, the need for technical support $(\bar{x}=1.51)$ and non-user friendly interface $(\bar{x}=1.53)$ were the major disadvantages for text-based mobile chat. The findings significantly revealed that additional effort is required to enhance text-based user interface by improving the consistency followed by navigation, simplicity and other factors.

Principally, preferences between TMC and GMC were consistent among all demographic background variables. Only minor difference tabulated in evaluating the user interface and usability for both services. The assessments between usability and user interface for the same service only differed marginally with mean value that was less than 0.2. The test subjects in the sample have assessed GMC favourably in both aspects of usability ( $\bar{x}=4.32)$ and user interface $(\bar{x}=4.37)$. On the contrary, the usability $(\bar{x}=1.62)$ and user interface $(\bar{x}=1.60)$ of the text-based mobile chat service was not gaining much support from the test subjects. Evidently, the research results elucidated that graphical-based usable mobile chats were perceived to have highly valued user interface which contributed to the usability of the service; whilst text-based user interface was not well appreciated to be a usable mobile chat.

The implications on mobile chat usability are noticeable. Research outcomes from the empirical evaluation were positive. In addition, the optimistic results typified the 
importance aspects and benefits that we could gain from employing a graphical-based usable interface on mobile chat. Conversely, in the effort to enhance the usability of text-based mobile chat, primary attention should be devoted in creating a user interface that promotes usages. This could be achieved by firstly understand the motives for the usage such as effectiveness, efficiency or satisfaction induced by the service. Although, no other significant relationship could be drawn to relate to the usability of the TMC, variables such as navigation ease and consistency of interface should not be quickly ruled out as trivial. This is due to the interpretation that upon invoking usage desire via the user interface, utilisation of the service might increase and subsequently, other variables should be given sufficient considerations.

As for the graphical-based usable mobile chat, usability could be augmented by proving user friendly interface that enhances user satisfaction, promotes usages and navigation ease. The findings significantly provided sufficient information for mobile chat designers to focus in enhancing the user interface and usability of mobile chat. Specifically, the needs of the target chatters should be identified to act as a framework for designing highly usable mobile chat that would consequently promote user satisfaction, user friendliness, usages and navigation ease.

\section{Conclusion}

Current mobile chat systems fail to replicate the success enjoyed by the static counterpart as the user interface design lacks in considering the mobile devices limitations. In particular, navigating through long chat archive in restricted screen display is cumbersome and time consuming. The effort to track a specific message sent by chatter includes scrolling through the archive and identifying each message in turn. The lack of usability in existing system motivated the needs to explore a novel interface to enhance the system effectiveness, efficiency and user satisfaction.

Although existing literature [5] cast pessimistic view on the future of mobile chat systems as the platform are deemed as not suitable for group communication, and novel interface that work around the device limitations could be a solution to promote mobile chat usages. The empirical evaluation outcomes corroborated to the research objectives and highlighted that the main difference between both services were the navigation ease with GMC leading significantly. Moreover, the research findings concluded that the user interface and usability of GMC were very well received unlike TMC which was perceived below average. On the contrary, TMC usability could be improved by creating user interface that encourages usages; whereas GMC usability is augmented by crafting user friendly interface that enhances user satisfaction, promotes usages and navigation ease. The used of specific components such as avatars, graphics and colours that constructed the graphical-based usable interface have drastically well addressed and improved the user satisfaction and navigation, and indirectly typified a strong linear relationship between user interface and usability of mobile chat. Additionally, the experimental results uncovered the major driving factors which include the user friendliness of interface that enhances user satisfaction, encourages usages and navigation ease extensively hold strong magnitude and correlated with the usability on GMC. 
In short, text-based mobile chat pales in comparison to that of the graphical-based counterpart. Moreover, every aspect of usability and user interface of the graphicalbased usable mobile chat is well appreciated by the test subjects. The empirical evaluation results have highlighted the potential use of graphical usable mobile chat as a substitution to the text-based chat that has poor reception and is under utilised in the commercial arena.

\section{References}

1. Chae, M., Kim, J.: What's so different about the mobile Internet? Communications of the ACM, pp. 240-247 (2003)

2. Vronay, D., Smith, M., Drucker, S.: Alternative interfaces for chat. In: Proceedings of the $12^{\text {th }}$ Annual ACM Symposium on User Interface Software and Technology, North Carolina, USA, pp. 19-26 (1999)

3. ISO/IEC JTC1 SC37.: Abstract collaborative workplace conceptual architecture contribution. [Online] (2004) Accessed on 18th November 2005 Available: WWW URL http://collab-tech.jtc1sc36.org/doc/SC36_WG2_N0077.pdf

4. Paelka, V., Reimann, C., Rosenbach, W.: A visualisation design repository for mobile devices. In: Proceedings of the $2^{\text {nd }}$ International Conference on Computer Graphics, Virtual Reality, Visualisation and Interaction, Cap Town, South Africa, pp. 57-62 (2003)

5. Burak, A., Sharon, T.: Usage patterns of FriendZone - mobile location-based community services. In: Proceedings of the $3^{\text {rd }}$ International Conference on Mobile and Ubiquitous Multimedia, Maryland, USA, pp. 93-100 (2004)

6. Grinter, R.E., Eldridge, M.A.: Y do tngrs luv 2 txt msg? Why "texting" became popular with teenagers. In: Proceedings of the $7^{\text {th }}$ European Conference on Computer Supported Cooperative Work (ECSCW), Bonn, Germany, Kluwer, pp. 219-238 (2001)

7. Grinter, R.E., Eldridge, M.: Wan2tlk?: Everyday text messaging. In: Proceedings of SIGCHI Conference on Human Factors in Computing Systems, Florida, USA, pp. 441448 (2003)

8. Viegas, F., Donath, J.: Chat circles. In: Proceedings of SIGCHI Conference on Human Factors in Computing Systems: the CHI is the limit, Pennsylvania, USA, pp. 9-16 (1999)

9. Viegas, F., Donath, J.: Chat circles series: Explorations in designing abstract graphical communication interfaces. In: Proceedings of the Conference on Designing Interactive Systems: Processes, Practices, Methods, and Techniques, London, England, pp. 359-369 (2002)

10. Hutchings, J.: Colour in Folklore and Tradition - The Principles. Colour Research and Application 29(1), 57-66 (2003)

11. Amin, A.K., Kersten, B.T.A., Kulyk, O.A., Pelgrim, P.H., Wang, C.M., Markopoulos, P.: SenseMS: A User-centered approach to enrich the messaging experience for teens by nonverbal means. In: Proceedings of the $7^{\text {th }}$ International Conference on Human Computer Interaction with Mobile Devices \& Services, Salzburg, Austria, pp. 161-166 (2005)

12. Berg, S., Taylor, A.S., Harper, R.: Mobile phones for the next generation: Device designs for teenagers. In: Proceedings of the SIGCHI Conference on Human Factors in Computing Systems, Florida, USA, pp. 433-440 (2003)

13. Persson, P.: ExMS: An animated and avatar-based messaging system for expressive peer communication. In: Proceedings of International ACM SIGGROUP Conference on Supporting Group Work, Florida, USA, pp. 31-39 (2003) 
14. Smith, M.A., Farnham, S.D., Drucker, S.M.: The social life of small graphical chat spaces. In: Proceedings of SIGCHI Conference on Human Factors in Computing Systems, The Hague, The Netherlands, pp. 462-469 (2000)

15. George, D., Mallery, P.: SPSS for Windows step by step: A simple guide and reference 10.0 update $3^{\text {rd }}$ edn., Allyn \& Bacon, Pearson Education Company, USA (2001)

16. Maxis Mobile.: Maxis SMS chat. [Online] (2002) Accessed on 23rd February 2006 Available: WWW URL http://store.maxis.com.my/pdf/SMSChat.pdf

17. Fox, S., Madden, M.: Generations online December 2005. [Online] (2005) Accessed on 27th March 2006 Available: WWW URL http://www.pewinternet.org/pdfs/ PIP_Generations_Memo.pdf

18. Lacey, M.: Multiple linear regression. [Online] (1997), Accessed on 23rd March 2006 Available: WWW URL http://www.stat.yale.edu/Courses/1997-98/101/linmult.htm 